\title{
Some numerical radius inequalities for Hilbert space operators
}

\author{
Mohsen Erfanian Omidvar, \\ Mohammad Sal Moslehian and Assadollah Niknam \\ (Communicated by Kenneth S. Berenhaut)
}

We present several numerical radius inequalities for Hilbert space operators. More precisely, we prove that if $A, B, C, D \in B(H)$ and $T=\left[\begin{array}{ll}A & B \\ C & D\end{array}\right]$ then $\max (w(A), w(D)) \leq \frac{1}{2}\left(\|T\|+\left\|T^{2}\right\|^{1 / 2}\right)$ and $\max \left((w(B C))^{1 / 2},(w(C B))^{1 / 2}\right) \leq$ $\frac{1}{2}\left(\|T\|+\left\|T^{2}\right\|^{1 / 2}\right)$. We also show that if $A \in B(H)$ is positive, then

$$
w(A X-X A) \leq \frac{1}{2}\|A\|\left(\|X\|+\left\|X^{2}\right\|^{1 / 2}\right) .
$$

\section{Introduction and preliminaries}

Let $B(H)$ denote the $C^{*}$-algebra of all bounded linear operators on a complex Hilbert space $H$ with inner product $\langle\cdot, \cdot\rangle$. For $A \in B(H)$ let

$$
\begin{aligned}
w(A) & =\sup \{|\langle x, A x\rangle|:\|x\|=1\}, \\
\|A\| & =\sup \{\|A x\|:\|x\|=1\}, \\
|A| & =\left(A^{*} A\right)^{1 / 2}
\end{aligned}
$$

denote the numerical radius, the usual operator norm of $A$ and the absolute value of $A$. It is well known that $w(\cdot)$ is a norm on $B(H)$, and that for all $A \in B(H)$,

$$
\frac{1}{2}\|A\| \leq w(A) \leq\|A\| .
$$

Here are some basic properties of the numerical radius:

$$
\begin{aligned}
w(|A|) & =\|A\|, \\
w\left(A^{*} A\right) & =w\left(A A^{*}\right), \\
w\left(U A U^{*}\right) & =w(A), \\
w\left(A_{1} \oplus A_{2} \oplus \cdots \oplus A_{n}\right) & =\max \left\{w\left(A_{i}\right): i=1,2, \ldots, n\right\},
\end{aligned}
$$

MSC2000: primary 47A62; secondary 46C15, 47A30, $15 \mathrm{~A} 24$.

Keywords: bounded linear operator, Hilbert space, norm inequality, numerical radius, positive operator. 
for all operators $A, A_{1}, A_{2}, \ldots, A_{n} \in B(H)$ and all unitary operators $U \in B(H)$.

Suppose $H=M_{1} \oplus M_{2}$ and $A \in B(H)$. Then we can write $A$ as a block matrix

$$
A=\left[\begin{array}{ll}
I_{1}^{*} A I_{1} & I_{1}^{*} A I_{2} \\
I_{2}^{*} A I_{1} & I_{2}^{*} A I_{2}
\end{array}\right],
$$

where $I_{i} \in B\left(M_{i}, H\right)$ such that $I_{i}(x)=x(i=1,2)$. If $A$ and $B$ are operators in $B(H)$ we write the direct sum $A \oplus B$ for the $2 \times 2$ operator matrix $\left[\begin{array}{cc}A & 0 \\ 0 & B\end{array}\right]$, regarded as an operator on $H \oplus H$. Thus

$$
\|A \oplus B\|=\left\|\left[\begin{array}{cc}
0 & A \\
B & 0
\end{array}\right]\right\|=\max (\|A\|,\|B\|) .
$$

Suppose $\mathscr{A}=A_{1} \oplus A_{2} \oplus \cdots \oplus A_{n}$, where $A_{i} \in B(H)$ and $x_{1}, x_{2}, \ldots, x_{n} \in H$. That is,

$$
\mathscr{A}=\left[\begin{array}{cccc}
A_{1} & 0 & \cdots & 0 \\
0 & A_{2} & \cdots & 0 \\
\vdots & \vdots & \ddots & \vdots \\
0 & 0 & \cdots & A_{n}
\end{array}\right]
$$

which we also write $\mathscr{A}=\operatorname{diag}\left(A_{1}, \ldots, A_{n}\right)$. Then

$$
\begin{gathered}
\left\langle\left[x_{1}, \ldots, x_{n}\right]^{T}, \mathscr{A}\left[x_{1}, \ldots, x_{n}\right]^{T}\right\rangle=\sum_{i=1}^{n}\left\langle x_{i}, A_{i}\left(x_{i}\right)\right\rangle, \\
w(\mathscr{A})=\sup \left\{\left|\left\langle\left[x_{1}, \ldots, x_{n}\right]^{T}, \mathscr{A}\left[x_{1}, \ldots, x_{n}\right]^{T}\right\rangle\right|: \sum_{i=1}^{n}\left\|x_{i}\right\|^{2}=1\right\} .
\end{gathered}
$$

For additional properties of the numerical radius, see [Bhatia 1997; Halmos 1982] and references therein.

Consider $A=\left[A_{i j}\right]$, where $A_{i j} \in B(H)$ and $i, j=1,2, \ldots, n$. We define $C(A)=A_{11} \oplus A_{22} \oplus \cdots \oplus A_{n n}$, called the $n$-pinching of $A$. We set $z=e^{2 \pi i / n}$ and $U:=\operatorname{diag}\left(I, z I, \ldots, z^{n-1} I\right)$, where $I$ is the identity operator in $B(H)$. Using the identity $\sum_{k=0}^{n-1} z^{k}=0$, one can see that $C(A)=(1 / n) \sum_{k=0}^{n-1} U^{* k} A U^{k}$ (see also [Bhatia 2000; 1997]).

It is shown in [Kittaneh 2005] that if $A, B, C, D, S, T \in B(H)$, then

$$
\begin{aligned}
w(A T B & +C S D) \\
& \leq \frac{1}{2}\left(\left\|A\left|T^{*}\right|^{2(1-\alpha)} A^{*}+B^{*}|T|^{2 \alpha} B+C\left|S^{*}\right|^{2(1-\alpha)} C^{*}+D^{*}|S|^{2 \alpha} D\right\|\right),
\end{aligned}
$$

for all $\alpha$ with $0 \leq \alpha \leq 1$. In particular, if $A, U, P \in B(H)$ such that $U$ is unitary 
and $P$ is projection, we have

$$
\begin{aligned}
w\left(A U \pm U^{*} A\right) & \leq \frac{1}{2}\left\||A|+\left|A^{*}\right|+U^{*}\left(|A|+\left|A^{*}\right|\right) U\right\| \leq\|A\|+\left\|A^{2}\right\|^{1 / 2}, \\
w(A P-P A) & \leq \frac{1}{2}\left\||A|+\left|A^{*}\right|+P\left(|A|+\left|A^{*}\right|\right) P\right\| \leq\|A\|+\left\|A^{2}\right\|^{1 / 2}, \\
w(A) & \leq \frac{1}{2}\left(\|A\|+\left\|A^{2}\right\|^{1 / 2}\right) .
\end{aligned}
$$

The last inequality refines the second inequality in (1-1); see also [Kittaneh 2003]. In [Kittaneh 2007; Bhatia and Kittaneh 2008] it is shown that if $A, B, X \in B(H)$ such that $A$ and $B$ are positive, then

$$
\|A X-X B|\|\leq \max (\|A\|,\|B\|)|\|X \mid\|,
$$

where $|\| \cdot|||$ is a unitarily invariant norm.

In particular,

$$
\|A X-X A\| \leq\|A\|\|X\| .
$$

In this paper we establish some inequalities sharper than inequalities (1-9) and $(1-11)$ to the numerical radius and we give a new proof of inequality (1-10). Some applications of these inequalities are considered as well.

\section{Main results}

In [Bhatia 1997] it is shown that

$$
\frac{1}{2}\|\|\left[\begin{array}{cc}
A+B & 0 \\
0 & A+B
\end{array}\right]\|\leq\|\left[\begin{array}{cc}
A & 0 \\
0 & B
\end{array}\right]\|\| \leq\left\|\left[\begin{array}{cc}
|A|+|B| & 0 \\
0 & 0
\end{array}\right]\right\|,
$$

where $|\| \cdot|||$ is a unitarily invariant norm. In this paper we extend this inequality to the numerical radius. We begin by establishing an interesting property of the numerical radius.

Lemma 2.1. Let $A \in B(H)$. Then

$$
w(C(A)) \leq w(A) .
$$

Proof. Since $C(A)=\frac{1}{n} \sum_{k=0}^{n-1} U^{* k} A U^{k}$, we have

$$
w(C(A)) \leq \frac{1}{n} \sum_{k=0}^{n-1} w\left(U^{* k} A U^{k}\right)=\frac{1}{n} \sum_{k=0}^{n-1} w(A)=w(A),
$$

where the inequality follows from property (1-4).

Theorem 2.2. Let $A_{1}, A_{2}, \ldots, A_{n} \in B(H)$. Then

$$
\frac{1}{n} w\left(\operatorname{diag}\left(\sum_{i=1}^{n} A_{i}, \ldots, \sum_{i=1}^{n} A_{i}\right)\right) \leq w(\mathscr{A}) \leq w\left(\operatorname{diag}\left(\sum_{i=1}^{n}\left|A_{i}\right|, 0, \ldots, 0\right)\right) .
$$


Proof. For the first inequality, we have, using (1-5),

$$
\begin{aligned}
w\left(\operatorname{diag}\left(\sum_{i=1}^{n} A_{i}, \ldots, \sum_{i=1}^{n} A_{i}\right)\right) & =w\left(\sum_{i=1}^{n} A_{i}\right) \leq \sum_{i=1}^{n} w\left(A_{i}\right) \\
& \leq n \max \left\{w\left(A_{i}\right): i=1,2, \ldots, n\right\}=n w(\mathscr{A}) .
\end{aligned}
$$

For the second inequality first we suppose $A_{1}, A_{2}, \ldots, A_{n}$ to be positive, so

$$
\begin{aligned}
w\left(\left[\begin{array}{cccc}
\sum_{i=1}^{n} A_{i} & 0 & \cdots & 0 \\
0 & 0 & \cdots & 0 \\
\vdots & \vdots & \ddots & \vdots \\
0 & 0 & \cdots & 0
\end{array}\right]\right)=w & \left(\left[\begin{array}{cccc}
A_{1}^{1 / 2} & A_{2}^{1 / 2} & \cdots & A_{n}^{1 / 2} \\
0 & 0 & \cdots & 0 \\
\vdots & \vdots & \ddots & \vdots \\
0 & 0 & \cdots & 0
\end{array}\right]\left[\begin{array}{cccc}
A_{1}^{1 / 2} & 0 & \cdots & 0 \\
A_{2}^{1 / 2} & 0 & \cdots & 0 \\
\vdots & \vdots & \ddots & \vdots \\
A_{n}^{1 / 2} & 0 & \cdots & 0
\end{array}\right]\right) \\
=w & \left(\left[\begin{array}{cccc}
A_{1}^{1 / 2} & 0 & \cdots & 0 \\
A_{2}^{1 / 2} & 0 & \cdots & 0 \\
\vdots & \vdots & \ddots & \vdots \\
A_{n}^{1 / 2} & 0 & \cdots & 0
\end{array}\right]\left[\begin{array}{cccc}
A_{1}^{1 / 2} & A_{2}^{1 / 2} & \cdots & A_{n}^{1 / 2} \\
0 & 0 & \cdots & 0 \\
\vdots & \vdots & \ddots & \vdots \\
0 & 0 & \cdots & 0
\end{array}\right]\right) \\
& =w\left(\left[\begin{array}{cccccc}
A_{1} & A_{1}^{1 / 2} A_{2}^{1 / 2} & \cdots & A_{1}^{1 / 2} A_{n}^{1 / 2} \\
A_{2}^{1 / 2} A_{1}^{1 / 2} & A_{2} & \cdots & A_{2}^{1 / 2} A_{n}^{1 / 2} \\
\vdots & & \ddots & \ddots & \vdots \\
A_{n}^{1 / 2} A_{1}^{1 / 2} & A_{n}^{1 / 2} A_{2}^{1 / 2} & \cdots & A_{n}
\end{array}\right]\right),
\end{aligned}
$$

where the second equality follows from (1-3). Using the inequality (2-1), we get

$$
\begin{aligned}
w\left(\left[\begin{array}{cccc}
A_{1} & 0 & \cdots & 0 \\
0 & A_{2} & \cdots & 0 \\
\vdots & \vdots & \ddots & \vdots \\
0 & 0 & \cdots & A_{n}
\end{array}\right]\right) & \leq w\left(\left[\begin{array}{cccc}
A_{1} & A_{1}^{1 / 2} A_{2}^{1 / 2} & \cdots & A_{1}^{1 / 2} A_{n}^{1 / 2} \\
A_{2}^{1 / 2} A_{1}^{1 / 2} & A_{2} & \cdots & A_{2}^{1 / 2} A_{n}^{1 / 2} \\
\vdots & \vdots & \ddots & \vdots \\
A_{n}^{1 / 2} A_{1}^{1 / 2} & A_{n}^{1 / 2} A_{2}^{1 / 2} & \cdots & A_{n}
\end{array}\right]\right) \\
& =w\left(\operatorname{diag}\left(\sum_{i=1}^{n} A_{i}, 0, \ldots, 0\right)\right)
\end{aligned}
$$

Now let $A_{1}, A_{2}, \ldots, A_{n}$ be arbitrary. Then

$$
w\left(\left[\begin{array}{cccc}
\left|A_{1}\right| & 0 & \cdots & 0 \\
0 & \left|A_{2}\right| & \cdots & 0 \\
\vdots & \vdots & \ddots & \vdots \\
0 & 0 & \cdots & \left|A_{n}\right|
\end{array}\right]\right) \leq w\left(\operatorname{diag}\left(\sum_{i=1}^{n}\left|A_{i}\right|, 0, \ldots, 0\right)\right) .
$$


Since

$$
w\left(\left[\begin{array}{cccc}
\left|A_{1}\right| & 0 & \cdots & 0 \\
0 & \left|A_{2}\right| & \cdots & 0 \\
\vdots & \vdots & \ddots & \vdots \\
0 & 0 & \cdots & \left|A_{n}\right|
\end{array}\right]\right)=w(|\mathscr{A}|) \geq w(\mathscr{A}),
$$

we have $w(\mathscr{A}) \leq w\left(\operatorname{diag}\left(\sum_{i=1}^{n}\left|A_{i}\right|, 0, \ldots, 0\right)\right)$.

Corollary 2.3. Let $A \in B(H)$. Then $\frac{1}{2} w\left(\left(A+A^{*}\right) \oplus\left(A+A^{*}\right)\right) \leq w\left(A \oplus A^{*}\right)$.

Kittaneh [2006] showed that if $A, B, C, D \in B(H)$ and if $T=\left[\begin{array}{ll}A & B \\ C & D\end{array}\right]$, then

$$
\max (r(A), r(D)) \leq \frac{1}{2}\left(\|T\|+\left\|T^{2}\right\|^{1 / 2}\right), \quad(r(B C))^{1 / 2} \leq \frac{1}{2}\left(\|T\|+\left\|T^{2}\right\|^{1 / 2}\right) .
$$

We show similar inequalities for the numerical radius. To achieve this, we need the following lemma [Kittaneh 2005].

Lemma 2.4. If $A, B \in B(H)$ and $A B=B A$, then $w(A B) \leq 2 w(A) w(B)$.

Theorem 2.5. If $A, B, C, D \in B(H)$ and $T=\left[\begin{array}{ll}A & B \\ C & D\end{array}\right]$, then

$$
\max (w(A), w(D)) \leq \frac{1}{2}\left(\|T\|+\left\|T^{2}\right\|^{1 / 2}\right),
$$

and

$$
\max \left((w(B C))^{1 / 2},(w(C B))^{1 / 2}\right) \leq \frac{1}{2}\left(\|T\|+\left\|T^{2}\right\|^{1 / 2}\right) .
$$

Proof.

By (1-5), we have $\max (w(A), w(D))=w\left(\left[\begin{array}{ll}A & 0 \\ 0 & D\end{array}\right]\right)$. Since $D$ is arbitrary,

$$
\max (w(A), w(D))=w\left(\left[\begin{array}{rr}
A & 0 \\
0 & -D
\end{array}\right]\right) .
$$

Consider the unitary operator $U=\left[\begin{array}{rr}I & 0 \\ 0 & -I\end{array}\right]$ on $H \oplus H$. Then $2\left[\begin{array}{rr}A & 0 \\ 0 & -D\end{array}\right]=T U+U T$. Thus

$$
\max (w(A), w(D)) \leq \frac{1}{2}\left(\|T\|+\left\|T^{2}\right\|^{1 / 2}\right),
$$

by inequality (1-8). This proves the inequality (2-2).

To prove the inequality (2-3), we note that

$$
\begin{aligned}
\max (w(B C), w(C B)) & =w\left(\left[\begin{array}{cc}
B C & 0 \\
0 & C B
\end{array}\right]\right) \quad(\text { by }(1-5)) \\
& =w\left(\left[\begin{array}{cc}
0 & B \\
C & 0
\end{array}\right]^{2}\right) \\
& \leq 2 w\left(\left[\begin{array}{cc}
0 & B \\
C & 0
\end{array}\right]\right)^{2} \quad \text { (by Lemma 2.4) }
\end{aligned}
$$


Since $B$ is arbitrary, we have

$$
\max (w(B C), w(C B)) \leq 2 w\left(\left[\begin{array}{rr}
0 & -B \\
C & 0
\end{array}\right]\right)^{2} .
$$

We observe that $2\left[\begin{array}{cc}0 & -B \\ C & 0\end{array}\right]=T U-U T$, so

$$
\max \left((w(B C))^{1 / 2},(w(C B))^{1 / 2}\right) \leq \frac{1}{2}\left(\|T\|+\left\|T^{2}\right\|^{1 / 2}\right)
$$

by inequality (1-8).

Corollary 2.6. If $A \in B(H)$, then

$$
w(A) \leq \frac{1}{2}\left(\|A\|+\left\|A^{2}\right\|^{1 / 2}\right) \leq\|A\| .
$$

Proof. Let $T=\left[\begin{array}{ll}A & 0 \\ 0 & 0\end{array}\right]$. Then

$$
\begin{array}{rlrl}
w(A) & \leq \frac{1}{2}\left(\|T\|+\left\|T^{2}\right\|^{1 / 2}\right) & & (\text { by }(2-2)) \\
& =\frac{1}{2}\left(\|A\|+\left\|A^{2}\right\|^{1 / 2}\right) & & (\text { by }(1-7)) \\
& \leq\|A\| . &
\end{array}
$$

Corollary 2.7. If $A \in B(H)$, then $\left\|A+A^{*}\right\| \leq\|A\|+\left\|A^{2}\right\|^{1 / 2} \leq 2\|A\|$.

Proof. Since $A+A^{*}$ is self-adjoint, we have

$$
\begin{aligned}
\frac{1}{2}\left\|A+A^{*}\right\| & =\frac{1}{2} w\left(\left(A+A^{*}\right) \oplus\left(A+A^{*}\right)\right) & & \text { (by (1-2) and (1-5)) } \\
& \leq w\left(A \oplus A^{*}\right) & & \text { (by Corollary 2.3) } \\
& \leq \frac{1}{2}\left(\left\|A \oplus A^{*}\right\|+\left\|\left(A \oplus A^{*}\right)^{2}\right\|^{1 / 2}\right) & & \text { (by Corollary 2.6) } \\
& =\frac{1}{2}\left(\|A\|+\left\|A^{2}\right\|^{1 / 2}\right) & & \text { (by (1-7)) } \\
& \leq\|A\| . & &
\end{aligned}
$$

We use some similar strategies as in [Kittaneh 2007] to prove the next two results.

Theorem 2.8. Let $A, P \in B(H)$ such that $P$ is a projection. Then

$$
w(A P-P A) \leq \frac{1}{2}\left(\|A\|+\left\|A^{2}\right\|^{1 / 2}\right) .
$$

Proof. Using the decomposition $H=\operatorname{ran} P \oplus \operatorname{ker} P$ and equality (1-6), we represent $P$ as the form $P=\left[\begin{array}{rr}I_{1} & 0 \\ 0 & 0\end{array}\right]$, where $I_{1}$ is the identity operator on $\operatorname{ran} P$. With respect to this decomposition, $A$ can be written as $A=\left[\begin{array}{ll}A_{11} & A_{12} \\ A_{21} & A_{22}\end{array}\right]$. Then

$$
P A-A P=\left[\begin{array}{cc}
0 & A_{12} \\
-A_{21} & 0
\end{array}\right]
$$


If $I_{2}$ is the identity operator on $\operatorname{ker} P$ and if $U=\left[\begin{array}{cc}I_{1} & 0 \\ 0 & -I_{2}\end{array}\right]$, then $U$ is unitary and $\left[\begin{array}{cc}0 & A_{12} \\ -A_{21} & 0\end{array}\right]=\frac{1}{2}(U A-A U)$. Therefore $w(A P-P A)=w\left(\left[\begin{array}{cc}0 & A_{12} \\ -A_{21} & 0\end{array}\right]\right)=\frac{1}{2} w\left(A U-U^{*} A\right) \leq \frac{1}{2}\left(\|A\|+\left\|A^{2}\right\|^{1 / 2}\right)$,

where the inequality follows from (1-8).

Theorem 2.9. Suppose that $A \in B(H)$ is positive. Then

$$
w(A X-X A) \leq \frac{1}{2}\|A\|\left(\|X\|+\left\|X^{2}\right\|^{1 / 2}\right) .
$$

Proof. First we prove that if $A$ is positive and a contraction, then

$$
w(A X-X A) \leq \frac{1}{2}\left(\|X\|+\left\|X^{2}\right\|^{1 / 2}\right) .
$$

If $R=\sqrt{A-A^{2}}$, the operator

$$
P=\left[\begin{array}{cc}
A & R \\
R & I-A
\end{array}\right]
$$

is a projection on $H \oplus H$, because $A \sqrt{A-A^{2}}=\sqrt{A-A^{2}} A$. If $Y=\left[\begin{array}{cc}X & 0 \\ 0 & 0\end{array}\right]$, then $P Y-Y P=\left[\begin{array}{cc}A X-X A & -X R \\ R X & 0\end{array}\right]$. By the inequality (2-4), we have

$$
w(Y P-P Y) \leq \frac{1}{2}\left(\|Y\|+\left\|Y^{2}\right\|^{1 / 2}\right) .
$$

Now if $Q=\left[\begin{array}{ll}I & 0 \\ 0 & 0\end{array}\right]$, then $\left[\begin{array}{cc}A X-X A & 0 \\ 0 & 0\end{array}\right]=Q(P Y-Y P) Q^{*}$, so

$$
\begin{aligned}
w\left(\left[\begin{array}{cc}
A X-X A & 0 \\
0 & 0
\end{array}\right]\right) & =w(Y P-P Y) & & (\text { by }(1-4)) \\
& \leq \frac{1}{2}\left(\|Y\|+\left\|Y^{2}\right\|^{1 / 2}\right) & & (\text { by }(2-4)) \\
& =\frac{1}{2}\left(\|X\|+\left\|X^{2}\right\|^{1 / 2}\right) & & (\text { by }(1-7))
\end{aligned}
$$

whence $w(A X-X A) \leq \frac{1}{2}\left(\|X\|+\left\|X^{2}\right\|^{1 / 2}\right)$. Let $A$ be a positive operator. It follows from the inequality

$$
w\left(\frac{A}{\|A\|} X-X \frac{A}{\|A\|}\right) \leq \frac{1}{2}\left(\|X\|+\left\|X^{2}\right\|^{1 / 2}\right)
$$

that $w(A X-X A) \leq \frac{1}{2}\|A\|\left(\|X\|+\left\|X^{2}\right\|^{1 / 2}\right)$.

Corollary 2.10. If $A, B \in B(H)$ such that $A$ is positive and $B$ is self-adjoint, then

$$
\|A B-B A\| \leq\|A\|\|B\| .
$$

Proof. The inequality (2-6) follows from (2-5) by letting $X=B$. 
Corollary 2.11. Suppose that $T \in B(H)$ has the cartesian decomposition $T=$ $A+i B$ such that $A$ is positive and $B$ is self-adjoint. Then

$$
\left\|T^{*} T-T T^{*}\right\| \leq\|A\|^{2}+\|B\|^{2} .
$$

Proof. By (2-6) and the arithmetic-geometric mean inequality, we have

$$
\left\|T^{*} T-T T^{*}\right\|=2\|A B-B A\| \leq 2\|A\|\|B\| \leq\|A\|^{2}+\|B\|^{2} .
$$

\section{References}

[Bhatia 1997] R. Bhatia, Matrix analysis, Grad. Texts in Math. 169, Springer, New York, 1997. MR 98i:15003 Zbl 0863.15001

[Bhatia 2000] R. Bhatia, "Pinching, trimming, truncating, and averaging of matrices", Amer. Math. Monthly 107:7 (2000), 602-608. MR 2001h:15020 Zbl 0984.15024

[Bhatia and Kittaneh 2008] R. Bhatia and F. Kittaneh, "Commutators, pinchings, and spectral variation”, Oper. Matrices 2:1 (2008), 143-151. MR 2392772 Zbl 1147.15019

[Halmos 1982] P. R. Halmos, A Hilbert space problem book, 2nd ed., Grad. Texts in Math. 19, Springer, New York, 1982. MR 84e:47001 Zbl 0496.47001

[Kittaneh 2003] F. Kittaneh, "A numerical radius inequality and an estimate for the numerical radius of the Frobenius companion matrix", Studia Math. 158:1 (2003), 11-17. MR 2004i:15022 Zbl 1113.15302

[Kittaneh 2005] F. Kittaneh, "Numerical radius inequalities for Hilbert space operators", Studia Math. 168:1 (2005), 73-80. MR 2005m:47009 Zbl 1072.47004

[Kittaneh 2006] F. Kittaneh, "Spectral radius inequalities for Hilbert space operators", Proc. Amer. Math. Soc. 134:2 (2006), 385-390. MR 2006d:47008 Zbl 1081.47010

[Kittaneh 2007] F. Kittaneh, "Inequalities for commutators of positive operators", J. Funct. Anal. 250:1 (2007), 132-143. MR 2008j:47031 Zbl 1131.47009

Received: 2009-05-05 Accepted: 2009-07-01

erfanian@mshdiau.ac.ir Department of Mathematics, Faculty of Science, Islamic Azad University-Mashhad Branch, Mashhad 91722, Iran

moslehian@ferdowsi.um.ac.ir Department of Pure Mathematics, Center of Excellence in Analysis on Algebraic Structures (CEAAS), Ferdowsi University of Mashhad, P.O. Box 1159, Mashhad 91775, Iran http://www.um.ac.ir/ moslehian

dassamankin@yahoo.co.uk

Department of Pure Mathematics, Center of Excellence in Analysis on Algebraic Structures (CEAAS), Ferdowsi University of Mashhad, P.O. Box 1159, Mashhad 91775, Iran 